\title{
New approaches beyond genetics: towards precision medicine in diabetes
}

\author{
Leif Groop ${ }^{1}$
}

Received: 20 April 2016/Accepted: 27 April 2016 / Published online: 8 October 2016

(C) Springer-Verlag Berlin Heidelberg 2016

Keywords ChIP sequencing - Classification - Diabetes · Epigenetics · Gene expression · Glucotoxicity · Pancreatic islets

In his State of the Union address in January 2015, President Obama launched an initiative to support research on precision medicine for diseases such as cancer and diabetes in the hope that personal information would significantly improve the healthcare and quality of life of patients. The field of cancer is much more advanced when it comes to precision medicine, and the field of diabetes has clearly been lagging behind. There are several reasons for this. The current classification of diabetes into two main forms is imprecise and poor in predicting disease outcome. A refined diabetes classification could provide a powerful tool to facilitate the implementation of individualised care from diagnosis in the same way as a genetic diagnosis of monogenic forms of diabetes guides clinicians to the optimal treatment [1]. This will require a much more comprehensive view than the current praxis of diagnosing diabetes based simply on measuring glucose. Diabetes results from a collision between a genetic predisposition and an affluent environment, and we need a more systematic approach to learn how this interaction leads to the disease. This could be achieved by combining some of the approaches presented in the symposium entitled 'New approaches beyond genetics' at the 2015 EASD annual meeting. The presentations included information on variation in the genome

Leif Groop

Leif.Groop@med.lu.se

1 Lund University Diabetes Centre, Jan Waldenströmsgata 35, 20505 Malmö, Sweden
(DNA) with variation in the expression of genes (RNA) and proteins in different tissues. In a related mini-review in this issue, Jerzy Adamski discusses how this is linked to a unique metabolite profile [2], while Bernd Mayer considers how it reflects the interaction between genome and environment [3]. The genetic information may not be stable, as epigenetic processes (DNA methylation, histone modifications, microRNAs, etc.) can induce reversible changes in the genome. Glucotoxicity is considered a central mechanism for deterioration of islet function and development of diabetic complications [4]. Furthermore, glucose is a strong trigger of histone modifications and, thereby, changes in gene expression, as exemplified by glucose-induced changes in the expression of the proinflammatory TXNIP gene in the kidney, which are mediated by histones [5]. Exploring glucotoxicity will require chromatin immunoprecipitation with massively parallel DNA sequencing (ChIP sequencing) of blood and tissues from hyperglycaemic individuals to identify the most glucose-sensitive genes and pathways.

While several groups have provided novel data on gene expression in adult human pancreatic islets [6-8] from patients with and without diabetes, this may not capture the whole process leading to beta cell dysfunction in type 2 diabetes, as a reduced beta cell mass could also be programmed during the fetal period, as reported for $K C N Q 1$. Expression of $K C N Q 1$ was normal in adult islets but methylated and imprinted in fetal islets [9]. Therefore, RNA sequencing of fetal islets should help to identify genes that might potentially be subject to imprinting during the intrauterine period [10]. We often assume that the same alleles confer the same risk when inherited from the mother and the father, but genes that are subject to imprinting often show a unique parent-of-origin transmission, which means that the maternal and paternal allele may have opposite effects [11]. 
Combining all this information can generate important biological information on disease pathogenesis, but will not explain why certain individuals are more prone to developing complications and or how a certain individual will respond to treatment - to do this we also need information on outcome, disease progression and treatment. Such information cannot be obtained from randomised controlled trials; instead, there is a wealth of unexplored information in patient records. We therefore need access to the health records of patients, including clinical chemistry measurements, diagnoses (ICD codes) and information from drug prescription registries. In the Nordic countries this is possible by linking the unique personal identifier, the person number, to such registries after institutional review board approval. Combining all available information to obtain a systematic picture of the disease (systems medicine) and linking this to detailed patient information will represent a first step towards precision medicine. This may not only improve the health and quality of life of patients, but also help the pharma industry to better stratify patients for drug trials and thereby reduce the costs associated with the development of new drugs.

Duality of interest The author declares that there is no duality of interest associated with this manuscript.

Contribution statement The author was the sole contributor to this paper.

\section{References}

1. Pearson E (2014) Stratified approaches to the management of diabetes. Diabet Med 31:393-398

2. Adamski J (2016) Key elements of metabolomics in the study of biomarkers of diabetes. Diabetologia doi:10.1007/s00125-0164044-y

3. Mayer B (2016) Using systems biology to evaluate targets and mechanism of action of drugs for diabetes comorbidities. Diabetologia doi:10.1007/s00125-016-4032-2

4. Brownlee M (2005) The pathobiology of diabetic complications: a unifying mechanism. Diabetes 54:1615-1625

5. De Marinis Y, Cai M, Bompada P et al (2016) Epigenetic regulation of the thioredoxin-interacting protein (TXNIP) gene by hyperglycemia in kidney. Kidney Int 89:342-353

6. Nica AC, Montgomery SB, Dimas AS et al (2010) Candidate causal regulatory effects by integration of expression QTLs with complex trait genetic associations. Plos Genet:e1000895

7. Taneera J, Lang S, Sharma A et al (2012) A systems genetics approach identifies novel genes and pathways for type 2 diabetes in human islets. Cell Metab 16:122-134

8. Fadista J, Vikman P, Ottosson Laakso E et al (2014) Global genomic and transcriptomic analysis of human pancreatic islets reveals novel genes influencing glucose metabolism. Proc Natl Acad Sci U S A 111:13924-13929

9. Travers ME, Mackay DJG, Dekker Nitert M et al (2013) Insights into the molecular mechanism for type 2 diabetes susceptibility at the $K C N Q 1$ locus from temporal changes in imprinting status in human islets. Diabetes 62:987-992

10. Prokopenko I, Poon W, Mägi R et al (2014) A central role for GRB10 in regulation of islet function in man. Plos Genet 10: 3e004235

11. Prasad RB, Lessmark A, Almgren P et al (2016) Excess maternal transmission of variants in the THADA gene to offspring with type 2 diabetes. Diabetologia. doi:10.1007/s00125-016-3973-9 